\title{
MicroRNA-495 serves as a diagnostic biomarker in patients with sepsis and regulates sepsis-induced inflammation and cardiac dysfunction
}

\author{
Hailei Guo ${ }^{1,2+}$, Liying Tang ${ }^{3 \dagger}$, Jianjun Xu ${ }^{1}$ Cai Lin ${ }^{1}$, Xiangwei Ling ${ }^{1}$, Caijiao Lu ${ }^{1 *}$ and Zhengjun Liu ${ }^{1 *}$
}

\begin{abstract}
Background: Sepsis leads to severe inflammatory and cardiac dysfunction. This study aimed to explore the clinical value of miR-495 in sepsis, as well as its role in sepsis-induced inflammation and cardiac dysfunction.

Methods: 105 sepsis patients were recruited; receiver operating characteristic (ROC) curve was plotted to assess the diagnostic value of miR-495 in sepsis. A model of sepsis in rats was created via performing cecal ligation and puncture (CLP). After modeling, the cardiac function, including left ventricular systolic pressure (LVSP), left ventricular end diastolic pressure (LVEDP) and maximum rate of rise/fall of left ventricle pressure ( $\pm \mathrm{dp} / \mathrm{dt}_{\text {max }}$ ), and serum cardiac troponin I (CTn-I), creative kinase isoenzyme MB (CK-MB) were detected. The blood cytokines levels including TNF- $a$, IL-6, IL-1 $\beta$ were also measured. Quantitative real-time PCR (qRT-PCR) was used for the measurement of the expression level of miR-495.

Results: MiR-495 was significantly downregulated in sepsis patients, especially patients who suffered from septic shock (SS). MiR-495 expression was negatively associated with Scr, WBC, CRP, PCT, APACHE II score and SOFA score. MiR-495 could distinguish patients with SS from non-SS patients. MiR-495 and SOFA score were better indictors for the occurrence of cardiac dysfunction in sepsis patients. In CLP-induced sepsis model. CLP rats experienced deterioration of LVSP, LVEDP, $\pm \mathrm{dp} / \mathrm{dt}_{\text {max }}$ and had a rise in serum CTn-I, CK-MB, TNF- $a$, IL-6 and IL-1 $\beta$, which were improved by miR-495 agomir injection.
\end{abstract}

Conclusions: MiR-495 might be a potential diagnostic biomarker for sepsis patients, and overexpression of miR-495 alleviated sepsis-induced inflammation and cardiac dysfunction.

Keywords: MiR-495, Sepsis, Diagnosis, Cardiac dysfunction, Inflammatory

\section{Background}

Sepsis is defined as the imbalance of the host response to infection, which can lead to life-threatening organ dysfunction [1]. Sepsis can lead to a severe systemic inflammatory response, even shock [2]. It has been reported that cardiac dysfunction is a major complication of

\footnotetext{
*Correspondence: jiaoos6710@163.com; liuzhjburn1977@163.com

${ }^{\dagger}$ Hailei Guo and Liying Tang contributed equally to this work

1 Department of Burns Surgery, The First Affiliated Hospital of Wenzhou Medical University, Nanbaixiang Street, Wenzhou 325000, Zhejiang,

People's Republic of China

Full list of author information is available at the end of the article
}

patients with sepsis, and is closely associated with sepsisinduced mortality [3]. Although major improvements have been made in critical care medicine in recent decades, the incidence and mortality of sepsis are still high $[4,5]$. Current studies focus on the identification of early biomarkers to predict sepsis outcome, including serum C-reactive protein (CRP), procalcitonin (PCT) levels [6], and acute physiology and chronic health evaluation II scores (APACHE II) and sequential organ failure assessment scores (SOFA) [7]. Novel biomarkers are beneficial for the early diagnosis and accurate assessment of sepsis. 
Furthermore, it may be helpful for fully understanding the mechanisms underlying sepsis.

MicroRNAs (miRNAs) are a kind of endogenous noncoding small RNAs with a length of approximately 22 nucleotides [8]. Currently, a large number of miRNAs have been identified to be potential novel biomarkers for early diagnosis or prognosis of various human diseases [9]. The use of miRNAs has also been widely reported to diagnose or stage sepsis in the critically ill [10-12]. Many reports demonstrated that miR-495 functions as a tumor suppressor, and modulates proliferation and migration of several tumor cells, such as colon cancer, gastric cancer, bladder cancer and so on [13-15]. Recent studies have already suggested the involvement of miR-495 in the inflammatory response of several human diseases, such as ankylosing spondylitis (AS), inflammatory bowel disease [16, 17]. Notably, Wang et al. [18] reported that miR495 is involved in the regulation of cardiac fibrosis, which plays a crucial role in sepsis-induced cardiac dysfunction. But the role of miR-495 in sepsis has not been reported.

Therefore, this study explored the expression and clinical role of miR-495 in sepsis, and further investigated its role in sepsis-induced inflammation and cardiac dysfunction.

\section{Materials and methods}

\section{Study population and sample collection}

This study was approved by the Committee on Ethics of the First Affiliated Hospital of Wenzhou Medical University. Written informed consent was obtained from all participants or their families. Following the Surviving Sepsis Campaign: International Guidelines for Management of Severe Sepsis and Septic Shock, 2012 [19], a total of 105 sepsis patients were recruited, who were admitted to intensive care units (ICUs) of the First Affiliated Hospital of Wenzhou Medical University from May 2016 to August 2017. Patients were excluded for any of the following reasons: less than 18 years old; in an immunocompromised state; pregnant; lactating; human immunodeficiency virus (HIV)-positive; in end-of-life conditions. All sepsis patients were further classified into septic shock (SS) group and non-septic shock (non-SS) group. SS patients were defined as sepsis patients who have persistent hypotension that requires vasopressors to maintain a $\mathrm{MAP} \geq 65 \mathrm{mmHg}$ and who have a serum lactate level $>2 \mathrm{mmol} / \mathrm{L}$ despite adequate volume resuscitation [20]. Among these sepsis patients, 71 were included as non-SS group, and other 34 patients were included in SS group. During treatment, the heart function of all patients was monitored and recorded. Additionally, 100 healthy age and gender-matched healthy individuals were collected as control group, who had no history of severe infection or malignancies, or other obvious abnormalities by physical examination. Within $24 \mathrm{~h}$ of admission to intensive care unit (ICU), the APACHE II score and SOFA score of sepsis patients were assessed, and blood samples were collected. The clinical data were recorded from each participant, including age, gender, body mass index (BMI), serum creatinine (Scr), albumin, white blood cell (WBC), CRP, PCT.

\section{Animal models of sepsis}

Male Sprague-Dawley rats weighing 250-300 g were purchased from the Laboratory Animal Center of Nanjing Medical University (Nanjing, Jiangsu Province, China), and housed under appropriate conditions $\left(25 \pm 2{ }^{\circ} \mathrm{C}\right.$ and $12 \mathrm{~h} \mathrm{light/dark} \mathrm{cycle)} \mathrm{with} \mathrm{free} \mathrm{access} \mathrm{to} \mathrm{water} \mathrm{and} \mathrm{food}$ before the experiment. All of the animal procedures were performed in accordance with the Guide for the Care and Use of Laboratory Animals and approved by the Medical Ethics Committee of the First Affiliated Hospital of Wenzhou Medical University. Cecal ligation and puncture (CLP) were performed to induce sepsis as described previously [21]. In brief, the rats were anesthetized with sodium pentobarbital $(50 \mathrm{mg} / \mathrm{kg}$, Sigma, St. Louis, MO, USA). A midline incision (about $2 \mathrm{~cm}$ ) was made on the anterior abdomen, then the cecum was exposed and the ligation was at the position of $30 \%$ of the cecum. Before closing the abdominal cavity, the cecum was punctured twice with a sterile 18-gauge needle to extrude the fecal material. The sham control rats underwent the same procedure without ligation and puncture. After surgery, 1-mL normal saline was injected to each rat for resuscitation.

\section{Animal grouping}

The rats were divided into four groups as follows: sham group (sham control rats, 1-mL normal saline was injected intravenously $24 \mathrm{~h}$ before the surgery); CLP group (CLP-treated rats, $1-\mathrm{mL}$ normal saline was injected intravenously $24 \mathrm{~h}$ before the surgery); miR495 negative control (NC) group (CLP-treated rats, $10 \mu \mathrm{g} \mathrm{miR}-495 \mathrm{NC}$ sequence was injected intravenously $24 \mathrm{~h}$ before the surgery); miR-495 agomir group (CLP-treated rats, $10 \mu \mathrm{g}$ miR-495 agomir was injected intravenously $24 \mathrm{~h}$ before the surgery). MiR-495 NC and miR-495 agomir were synthesized and provided by GenePharma, Shanghai, China. After molding and injection, each group had at least 8 viable individuals.

\section{Cardiac function and blood cytokines assessments}

After surgery, the cardiac function of rats was detected. The LVSP, LVEDP and maximum rate of rise/fall of left ventricle pressure $\left( \pm \mathrm{dp} / \mathrm{dt}_{\max }\right)$ were measured using the MFLab 3.01 package in FDP-1 HRV \& BRS 
analysis system (Shanghai Jialong, Shanghai, China). 5 -mL venous blood sample was collected from each rat. Enzyme-linked immunosorbent assay (ELISA) was performed to measure the content of serum cardiac troponin I (CTn-I) and creative kinase isoenzyme $\mathrm{MB}$ (CK-MB), and serum levels of tumor necrosis factor alpha (TNF- $\alpha)$, interleukin 6 (IL-6), IL-1 $\beta$.

\section{RNA extraction and quantitative real-time polymerase chain reaction (qRT-PCR)}

After all the tests were finished, the rats were killed and the tissues from myocardium were collected. Total RNA was isolated using Trizol reagent (Life Technologies Corporation, Carlsbad, CA, USA). TaqMan miRNA reverse transcription kit (Applied Biosystems, Foster City, CA, USA) was used for reverse transcription. The Real-Time PCR analysis was performed using the One Step SYBR ${ }^{\circledR}$ PrimeScript ${ }^{\circledR}$ PLUS RT-RNA PCR Kit (TaKaRa Biotechnology, Dalian, China). The relative expression of miR-495 was determined in relation to U6 by the comparative delta CT $\left(2^{-\Delta \Delta C t}\right)$ method.

\section{Statistical analysis}

All data analyses were conducted using SPSS version 18.0 software (SPSS Inc., Chicago, IL) and GraphPad Prism 5.0 software (GraphPad Software, Inc., USA). The data were expressed as mean \pm standard deviation (SD). Differences between groups were compared using the Chi-square test, student's $t$ test or one-way ANOVA analysis. Correlations were determined by Spearman's rank correlation test. Binary logistic regression analysis was used to evaluate the association of different variables with the occurrence of cardiac dysfunction in sepsis patients. Receiver operating characteristic (ROC) curve was plotted, and the area under curve (AUC) with $95 \%$ confidence interval (CI) was calculated to assess the discrimination ability of miR-495 expression between sepsis patients and healthy controls. $P<0.05$ was considered to indicate a statistically significant difference.

\section{Results}

\section{Clinical characteristics of study objects}

There were 100 healthy controls and 105 sepsis patients with the mean age of $56.39 \pm 10.20$ and $55.06 \pm 9.17$, respectively (Table 1 ). No significant difference was detected for age, gender and BMI between the two groups (all $P>0.05$ ). The serum levels of Scr, WBC, CRP and PCT were higher in sepsis patients than that in healthy controls, whereas the serum albumin level was lower in patients group, and all differences reached
Table 1 Comparison of the baseline data between the two groups of study objects

\begin{tabular}{llll}
\hline Parameters & $\begin{array}{l}\text { Health } \\
(\mathbf{n}=100)\end{array}$ & $\begin{array}{l}\text { Sepsis } \\
(\mathbf{n}=\mathbf{1 0 5})\end{array}$ & P value \\
\hline Age (year) & $56.39 \pm 10.20$ & $55.06 \pm 9.17$ & 0.327 \\
Gender (male/female) & $61 / 39$ & $59 / 46$ & 0.485 \\
BMI (kg/m $\left.{ }^{2}\right)$ & $20.54 \pm 1.87$ & $20.52 \pm 1.97$ & 0.955 \\
Scr $(\mathrm{mg} / \mathrm{dL})$ & $1.10 \pm 0.32$ & $1.69 \pm 0.43$ & $<0.001$ \\
Albumin $(\mathrm{g} / \mathrm{L})$ & $40.74 \pm 4.37$ & $25.69 \pm 4.34$ & $<0.001$ \\
WBC $\left(\times 10^{9} / \mathrm{L}\right)$ & $7.53 \pm 1.61$ & $18.73 \pm 6.29$ & $<0.001$ \\
CRP $(\mathrm{mg} / \mathrm{L})$ & $6.03 \pm 2.46$ & $94.90 \pm 26.23$ & $<0.001$ \\
PCT $(\mathrm{ng} / \mathrm{mL})$ & $0.05 \pm 0.02$ & $12.88 \pm 5.24$ & $<0.001$ \\
APACHE II score & - & $12.92 \pm 3.22$ & - \\
SOFA score & - & $5.28 \pm 1.31$ & - \\
\hline
\end{tabular}

$B M I$ body mass index, $S c r$ serum creatinine, $W B C$ white blood cell, $C R P C$-reactive protein, $P C T$ procalcitonin, APACHE acute physiology and chronic health evaluation, SOFA sequential organ failure assessment

significant levels (all $P<0.001$ ). And the mean value of APACHE II score and SOFA score in sepsis patients were $12.92 \pm 3.22$ and $5.28 \pm 1.31$, respectively.

\section{Serum level of miR-495 in sepsis patients}

The serum expression level of miR-495 in sepsis patients and healthy controls were measured using qRT-PCR. As shown in Fig. 1a, serum miR-495 was significantly downregulated in patients group compared with healthy controls $(P<0.001)$. Additionally, we further compared the miR-495 expression levels between non-SS and SS patients. It was found that miR-495 level in SS group was significantly lower than that in non-SS group (Fig. 1b, $P<0.001)$.

\section{Correlation of miR-495 expression with clinicopathological features of sepsis patients}

Regarding all the clinicopathological features in sepsis patients, miR-495 expression was negatively associated with Scr, WBC, CRP, PCT, APACHE II score and SOFA score (all $P<0.05$, Table 2). However, miR-495 expression was not associated with age, gender, BMI and albumin (all $P>0.05$ ). These results indicated that serum miR-495 expression level was negatively associated with severity in sepsis patients.

\section{Diagnostic value of miR-495 expression for patients with sepsis}

The ROC curve was conducted to assess the diagnostic value of miR-495 in sepsis with the serum miR-495 expression in sepsis patients, and corresponding healthy individuals were used as control. As shown in Fig. 2a, the diagnostic value of serum miR-495 level in sepsis patients compared with healthy controls was assessed. It was found 

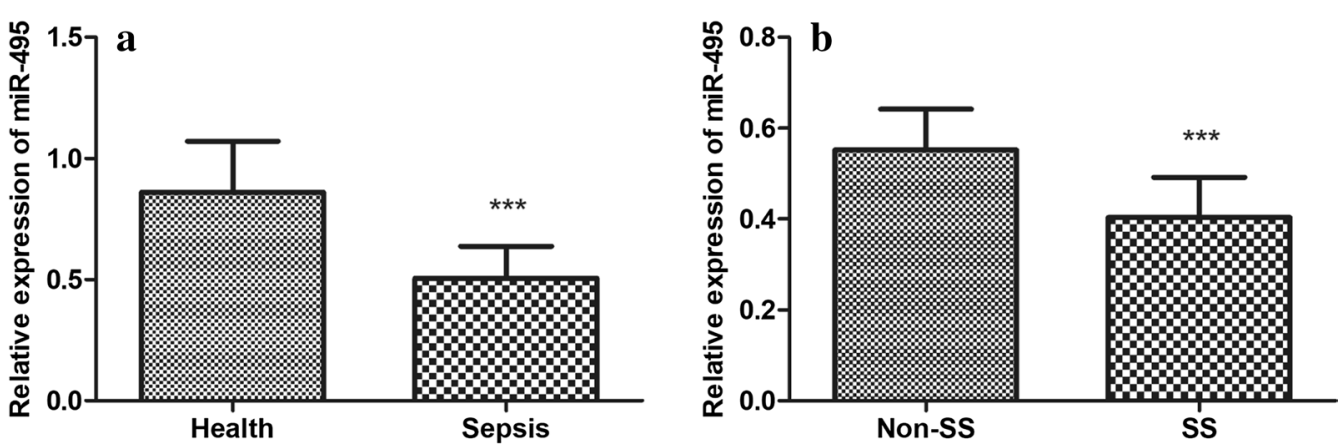

Fig. 1 The serum expression level of miR-495 in sepsis patients and healthy controls were measured using qRT-PCR. a MiR-495 was significantly downregulated in patients group compared with healthy controls. b MiR-495 level in SS group was significantly lower than that in non-SS group. *** $P<0.001$

Table 2 Correlation of miR-495 relative expression with clinical characteristics

\begin{tabular}{|c|c|c|}
\hline \multirow[t]{2}{*}{ Parameters } & \multicolumn{2}{|c|}{ MiR-495 expression } \\
\hline & $P$ value & $\begin{array}{l}\text { Correlation } \\
\text { coefficient }(r)\end{array}$ \\
\hline Age & 0.902 & 0.012 \\
\hline Gender & 0.519 & -0.064 \\
\hline $\mathrm{BMI}$ & 0.435 & 0.077 \\
\hline $\mathrm{Scr}$ & 0.035 & -0.206 \\
\hline Albumin & 0.192 & 0.128 \\
\hline WBC & $<0.001$ & -0.573 \\
\hline CRP & $<0.001$ & -0.509 \\
\hline PCT & $<0.001$ & -0.404 \\
\hline APACHE II score & $<0.001$ & -0.500 \\
\hline SOFA score & $<0.001$ & -0.523 \\
\hline
\end{tabular}

$B M I$ body mass index, Scr serum creatinine, WBC white blood cell, $C R P C$-reactive protein, $P C T$ procalcitonin, APACHE acute physiology and chronic health evaluation, SOFA sequential organ failure assessment

that the AUC value was 0.915 yielding the sensitivity of $89.5 \%$ and specificity of $83.0 \%$ at the cutoff value of 0.655 . These results indicated that serum miR-495 levels could be used to distinguish sepsis patients from healthy individuals. Additionally, the diagnostic value of miR-495 level in SS patients compared with non-SS patients was further evaluated. As shown in Fig. 2b, the AUC value was 0.885 yielding the sensitivity of $85.3 \%$ and specificity of $87.3 \%$ at the cutoff value of 0.475 . It indicated that miR-495 could distinguish patients with SS from non-SS patients.

\section{Association of miR-495 expression with the occurrence of cardiac dysfunction in sepsis patients}

According to the results of heart function monitor, the sepsis patients were divided into two groups: the normal group $(\mathrm{n}=39)$ and the cardiac dysfunction group $(n=66)$. Then, the logistic regression analysis was performed to evaluate the association miR-495 expression with the occurrence of cardiac dysfunction. As shown in Table 3, it was observed that downregulation of miR$495(\mathrm{OR}=0.237,95 \% \mathrm{CI} 0.082-0.689, P=0.08)$ and high level of SOFA score $(\mathrm{OR}=3.291,95 \%$ CI $1.267-8.552$, $P=0.014$ ) were independent factors for the occurrence of cardiac dysfunction in sepsis patients. It was concluded that miR-495 and SOFA scores were better indictors for the occurrence of cardiac dysfunction in sepsis patients.

\section{MiR-495 regulates sepsis-induced cardiac dysfunction in rat models}

To investigate the potential role of miR-495 in sepsis-induced cardiac dysfunction, sepsis model was constructed in rats. As shown in Fig. 3a, b, miR-495 expression was significantly downregulated in both rat serum and myocardium after CLP modeling, which was reversed by miR- 495 agomir injection (all $P<0.01$ ). It was suggested that miR-495 agomir injection could effectively upregulate miR-495 expression in sepsis rats. Furthermore, the cardiac function of rats was assayed. It was found that LVSP and $+\mathrm{dp} / \mathrm{dt}_{\max }$ significantly decreased, whereas LVEDP and $-\mathrm{dp} / \mathrm{dt}_{\max }$ remarkably increased in the CLP group compared with the sham group (all $P<0.01$, Fig. 3c-e). Additionally, serum cTnI, CK-MB also showed remarkably increasing trend in the CLP group compared with the sham group (all $P<0.01$, Fig. 3f, g). These results suggested that myocardial dysfunction occurred in the rat sepsis model. However, it was noted that overexpression of miR-495 significantly reversed the effect of CLP surgery on myocardial function of rats, which was indicated by the increase of LVSP and $+\mathrm{dp} /$ $\mathrm{dt}_{\max }$ and the decrease of LVEDP and $-\mathrm{dp} / \mathrm{dt}_{\max }$, as well as serum cTnI, CK-MB levels. We concluded that miR495 may be involved in the regulation of myocardial function of sepsis. 

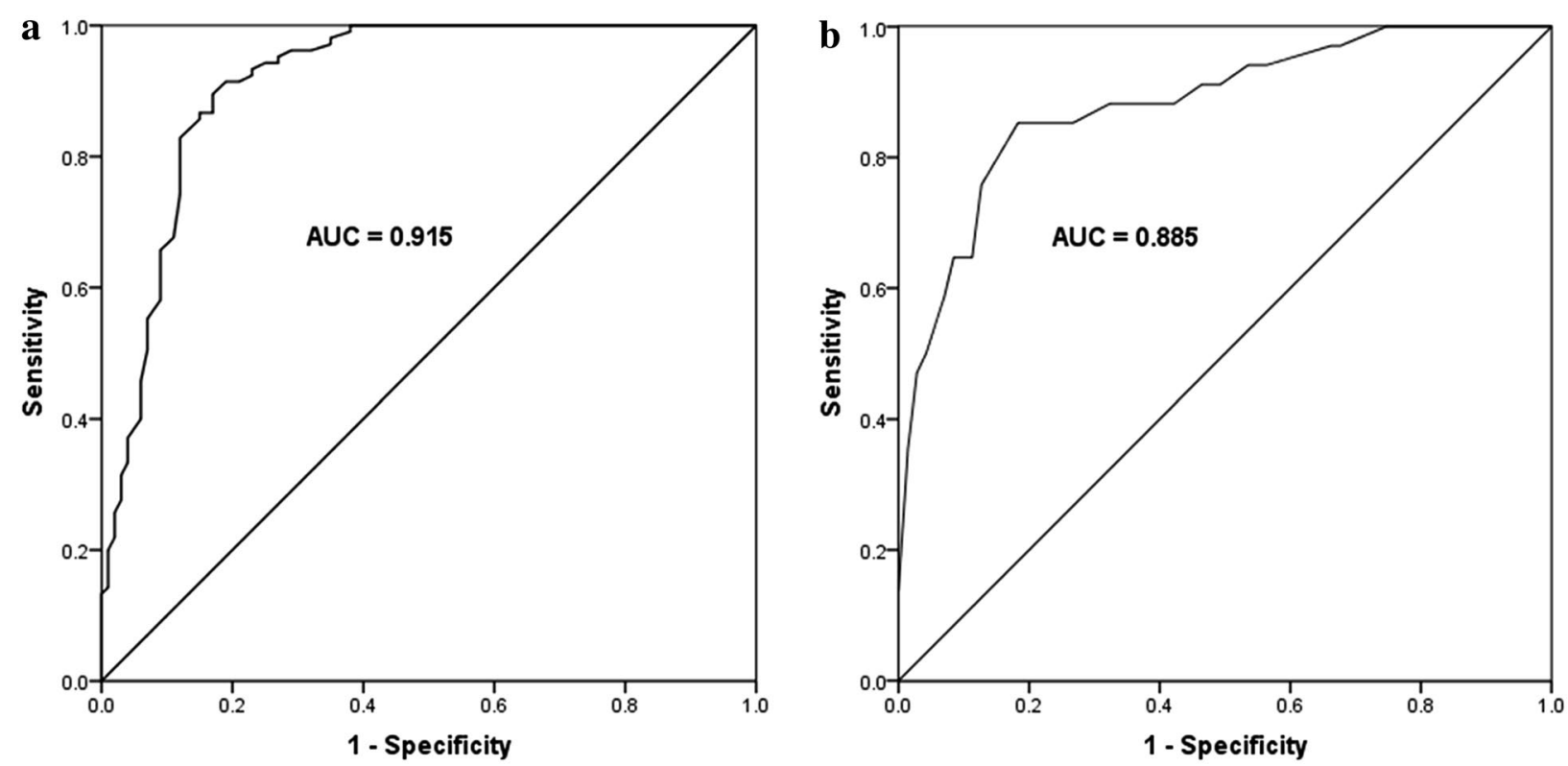

Fig. 2 Receiver operating characteristic (ROC) curve for patients with sepsis and healthy controls. a The diagnostic value of serum miR-495 level in sepsis patients compared with healthy controls. The area under curve (AUC) value was 0.915 yield the sensitivity of $89.5 \%$ and specificity of $83.0 \%$ at the cutoff value of 0.655 . b The diagnostic value of miR-495 level in SS patients compared with non-SS patients. The AUC value was 0.885 yield the sensitivity of $85.3 \%$ and specificity of $87.3 \%$ at the cutoff value of 0.475

Table 3 Association of different variables with the occurrence of heart dysfunction in sepsis patients

\begin{tabular}{llll}
\hline Variables & OR & $\mathbf{9 5 \%} \mathbf{C l}$ & P value \\
\hline MiR-495 & 0.237 & $0.082-0.689$ & 0.008 \\
Age & 1.050 & $0.440-2.505$ & 0.912 \\
Gender & 1.198 & $0.494-2.906$ & 0.689 \\
BMl & 1.244 & $0.442-3.505$ & 0.679 \\
SCr & 1.090 & $0.453-2.623$ & 0.848 \\
Albumin & 0.726 & $0.282-1.869$ & 0.507 \\
WBC & 0.749 & $0.249-2.259$ & 0.608 \\
CRP & 1.289 & $0.472-3.520$ & 0.621 \\
PCT & 2.199 & $0.717-6.751$ & 0.168 \\
APACHE II score & 1.978 & $0.757-5.163$ & 0.164 \\
SOFA score & 3.291 & $1.267-8.552$ & 0.014 \\
\hline
\end{tabular}

$B M I$ body mass index, Scr serum creatinine, WBC white blood cell, CRP C-reactive protein, $P C T$ procalcitonin, APACHE acute physiology and chronic health evaluation, SOFA sequential organ failure assessment

\section{MiR-495 regulates sepsis-induced inflammation in rat models}

To explore the effect of miR-495 on sepsis-induced inflammation, the levels of three inflammatory factors in rat serum were detected. It was observed that serum TNF- $\alpha$, IL-6 and IL-1 $\beta$ levels were all increased significantly in CLP group compared with the sham group, which were effectively attenuated by miR-495 agomir injection (all $P<0.05$, Fig. 4). These results indicated that
CLP-induced sepsis presented obvious inflammatory reaction, but miR-495 overexpression could relive sepsisinduced inflammation.

\section{Discussion}

Sepsis is a relatively common SIRS in the intensive care unit (ICU), and has a high rate of fatality. The immediate diagnosis and intervention are beneficial for the satisfactory clinical outcomes of sepsis patients. A growing number of researchers are applying themselves to identify the biomarkers for the early diagnosis of sepsis. Currently, PCT is approved to be used as a biomarker to differentiate culture-negative and culture-positive sepsis from non-infectious SIRS [22]. Additionally, several other diagnostic and prognostic biomarkers have been identified in sepsis, such as CRP, serum lactate, and IL-6, but the sensitivity or specificity is not high enough for enhancing the timely intervention of sepsis [23-25].

Dysregulated miRNA expression has been widely reported to be associated with the clinical features of SIRS, critically ill polytrauma, and sepsis [26, 27]. In the present study, miR-495 was determined to be downregulated in sepsis patients for the first time. Previous study has suggested that miR-495 is an important miRNA involved in multiple kinds of cancers, and is also associated with the proliferation, invasion, metastasis and drug resistance of tumor cells. For example, in endometrial cancer (EC), miR-495 was downregulated 


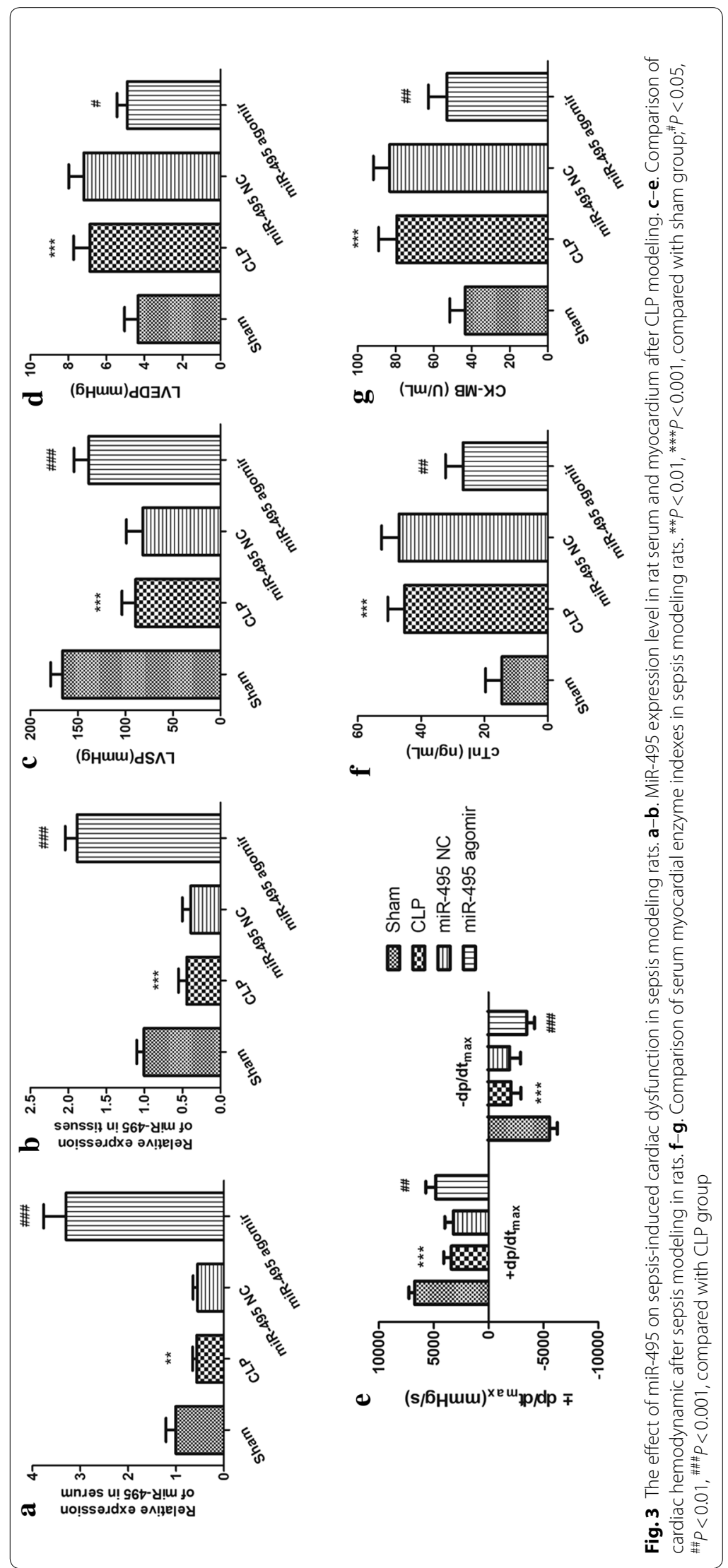



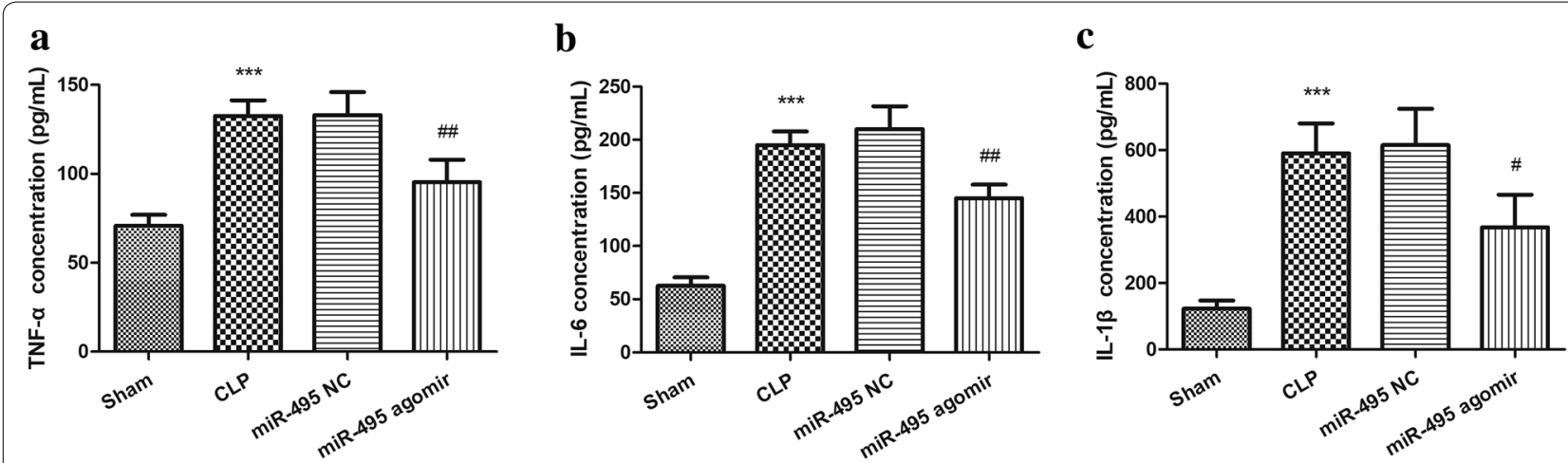

Fig. 4 Serum indexes of inflammatory response after sepsis modeling in rats. ${ }^{* * *} P<0.001$, compared with sham group; ${ }^{\#} P<0.05$, ${ }^{\# \#} P<0.01$, compared with CLP group

in tumor tissues, and overexpression of miR-495 markedly inhibited tumor cell proliferation and promoted cell apoptosis via targeting PIK3R1 [28]. In oral squamous cell carcinoma (OSCC), miR-495 was also reported to play an antitumor effect through regulating OSCC cell growth and metastasis [29]. In healthy cells, miR-495 was also reported to participate in a variety of developmental, apoptotic, immune and inflammatory mechanisms [30]. Additionally, miR-495 has also been identified to be a diagnostic or prognostic biomarker in several diseases, and might be a potential therapeutic target for the treatment of diseases [31-33]. Our study suggested that serum miR-495 expression level showed close correlation with several inflammatory marker in sepsis patients, and ROC curve analysis suggested serum miR-495 to be a potential diagnostic biomarker to distinguish sepsis patients from healthy individuals. Furthermore, miR495 was also overexpressed in SS patients, and miR-495 could distinguish patients with SS from non-SS patients. We concluded that miR-495 might be associated with the severity of sepsis.

Previous study has demonstrated that cardiac dysfunction is a major complication in sepsis patients, and is closely related to sepsis-induced mortality [34]. Approximately, half of the patients with severe sepsis suffer myocardial depression to some extent, with the high mortality of $70-90 \%$ [35]. MiR-495 has been widely reported to play a crucial role in various cardiac diseases, and involved in the regulation of myocardial function $[18,36]$. Notably, miR-495 is involved in the regulation of cardiac fibrosis, which plays a crucial role in sepsis-induced cardiac dysfunction [18, 37]. In the present study, logistic regression analysis was performed to evaluate the association miR-495 expression with the occurrence of cardiac dysfunction. It was observed that downregulation of miR-495 and high level of SOFA score were independent factors for the occurrence of cardiac dysfunction in sepsis patients. Furthermore, we established a sepsis model in rats to explore the role of miR-495 in sepsis-induced cardiac dysfunction. Our study showed that the sepsis model in rats developed severe cardiac dysfunction, but it was significantly improved by miR-495 upregulating, which was consistent with the previous evidences. We concluded that miR-495 may be involved in the regulation of myocardial function of sepsis.

MiR-495 is an inflammatory-related miRNA and has been reported to play an anti-inflammatory role in several human diseases. Du et al. [16] reported that the level of miR-495 was decreased in AS patients, and the cell experiment suggested that miR-495 suppressed the inflammatory response of AS by downregulating TNF- $\alpha$, IL-1 $\beta$ and IL-6. Another study about cardiac microvascular endothelial cell (CMEC) injury reported that miR-495 ameliorated CMEC injury and inhibited inflammatory reaction via suppressing the NLRP3 inflammasome signaling pathway, and NLRP3 was proved to be the target gene of miR-495 [36]. Consistent with the previous evidence, the present study results demonstrated that the CLP rats had a significant rise in serum TNF- $\alpha$, IL- 6 and IL-1 $\beta$, which were dropped by miR-495 overexpression. It is well known that these inflammatory factors are major contributors to the development of cardiac dysfunction in sepsis $[38,39]$. These results accompany with the previous evidence, we concluded that miR-495 plays a significant role against the inflammatory response, and the anti-inflammatory effect of miR-495 might be associated with its cardioprotective effects on sepsis-induced cardiac dysfunction. However, there were some limitations in our study. The study population was relatively small, and a much larger group is needed to verify the present results. Additionally, further studies are warranted to investigate the concrete mechanism underlying the role of miR-495 in heart function of sepsis. 
In summary, our experiments demonstrated that miR495 was downregulated in sepsis patients, and was a better indicator for the occurrence of cardiac dysfunction in sepsis patients. Overexpression of miR-495 alleviated sepsisinduced inflammation and cardiac dysfunction. MiR-495 might be a potential diagnostic biomarker and will facilitate early treatment of sepsis.

\section{Abbreviations}

CLP: cecal ligation and puncture; LVSP: left ventricular systolic pressure; LVEDP: left ventricular end diastolic pressure; CTn-I: cardiac troponin I; GRT-PCR: quantitative real-time PCR; CK-MB: creative kinase isoenzyme MB; SIRS: systemic inflammatory response syndrome; CRP: C-reactive protein; PCT: procalcitonin; APACHE II: acute physiology and chronic health evaluation II scores; SOFA: sequential organ failure assessment scores; miRNAs: MicroRNAs; AS: ankylosing spondylitis; ICUs: intensive care units; HIV: human immunodeficiency virus; BMI: body mass index; Scr: serum creatinine; WBC: albumin, white blood cell; CLP: cecal ligation and puncture; NC: negative control; ELISA: enzyme-linked immunosorbent assay; TNF-a: tumor necrosis factor alpha; IL-6: interleukin 6; qRT-PCR: quantitative real-time polymerase chain reaction; SD: standard deviation; ROC: receiver operating characteristic; AUC: the area under curve; $\mathrm{Cl}$ : confidence interval; CMEC: cardiac microvascular endothelial cell.

\section{Acknowledgements}

Not applicable.

\section{Authors' contributions}

HG and LT made substantial contributions to conception and design, analysis and interpretation of data, preparation of manuscript. JX, CL and XL contributed to acquisition of data, and experiments. CL and ZL contributed to conception and design, and revision of manuscript. All authors read and approved the final manuscript.

\section{Funding}

No funding was received.

\section{Availability of data and materials}

All data generated during this study are included in this published article.

\section{Ethics approval and consent to participate}

This study was approved by the Committee on Ethics of the First Affiliated Hospital of Wenzhou Medical University. Written informed consent was obtained from all participants or their families.All of the animal procedures were performed in accordance with the Guide for the Care and Use of Laboratory Animals and approved by the Medical Ethics Committee of the First Affiliated Hospital of Wenzhou Medical University.

\section{Consent for publication}

Not applicable.

\section{Competing interests}

The authors declare that they have no competing interests.

\section{Author details}

${ }^{1}$ Department of Burns Surgery, The First Affiliated Hospital of Wenzhou Medical University, Nanbaixiang Street, Wenzhou 325000, Zhejiang, People's Republic of China. ${ }^{2}$ Department of Burns Surgery, Jinan Central Hospital Affiliated to Shandong University, Jinan 250013, People's Republic of China. ${ }^{3}$ Department of Rehabilitation, Wenzhou Hospital of Traditional Chinese Medicine Affiliated to Zhejiang, Chinese Medical University, Wenzhou 32500, People's Republic of China.

Received: 31 July 2019 Accepted: 11 November 2019 Published online: 26 November 2019

\section{References}

1. Baumann S. Cytarabine, venous catheter removal, sepsis, diagnosis of malignancy, and takotsubo syndrome. Oncol Res Treat. 2015;38(3):125.

2. Zhu C, Chen T, Liu B. Inhibitory effects of miR-25 targeting HMGB1 on macrophage secretion of inflammatory cytokines in sepsis. Oncol Lett. 2018;16(4):5027-33.

3. Martin L, Derwall M, Thiemermann C, SchurholzT. Heart in sepsis: molecular mechanisms, diagnosis and therapy of septic cardiomyopathy. Anaesthesist. 2017:66(7):479-90.

4. Chen X, Tong H, Chen Y, Chen C, Ye J, Mo Q, et al. Klotho ameliorates sepsis-induced acute kidney injury but is irrelevant to autophagy. Onco Targets Ther. 2018;11:867-81.

5. Jin $Y H$, Li ZT, Chen $H$, Jiang $X Q$, Zhang YY, Wu F. Effect of dexmedetomidine on kidney injury in sepsis rats through TLR4/MyD88/NF-kappaB/ iNOS signaling pathway. Eur Rev Med Pharmacol Sci. 2019;23(11):5020-5.

6. Hillas G, Vassilakopoulos T, Plantza P, Rasidakis A, Bakakos P. C-reactive protein and procalcitonin as predictors of survival and septic shock in ventilator-associated pneumonia. Eur Respir J. 2010;35(4):805-11.

7. Chen SJ, Chao TF, Chiang MC, Kuo SC, Chen LY, Yin T, et al. Prediction of patient outcome from Acinetobacter baumannii bacteremia with sequential organ failure assessment (SOFA) and acute physiology and chronic health evaluation (APACHE) II scores. Intern Med. 2011;50(8):871-7.

8. Huang D, Wang F, Wu W, Lian C, Liu E. MicroRNA-429 inhibits cancer cell proliferation and migration by targeting the AKT1 in melanoma. Cancer Biomark. 2019. https://doi.org/10.3233/CBM-190289.

9. Chen X, Ba Y, Ma L, Cai X, Yin Y, Wang K, et al. Characterization of microRNAs in serum: a novel class of biomarkers for diagnosis of cancer and other diseases. Cell Res. 2008;18(10):997-1006.

10. Xie J, Zhang L, Fan X, Dong X, Zhang Z, Fan W. MicroRNA-146a improves sepsis-induced cardiomyopathy by regulating the TLR-4/NF-kappaB signaling pathway. Exp Ther Med. 2019;18(1):779-85.

11. Vincent $\mathrm{J}$. The clinical challenge of sepsis identification and monitoring. PLoS Med. 2016;13(5):e1002022.

12. Rhodes A, Evans LE, Alhazzani W, Levy MM, Antonelli M, Ferrer R, et al. Surviving sepsis campaign: international guidelines for management of sepsis and septic shock: 2016. Intensive Care Med. 2017;43(3):304-77.

13. He Z, Dang J, Song A, Cui X, Ma Z, Zhang Z. NEAT1 promotes colon cancer progression through sponging miR-495-3p and activating CDK6 in vitro and in vivo. J Cell Physiol. 2019. https://doi.org/10.1002/jcp.28557.

14. Chen S, Wu J, Jiao K, Wu Q, Ma J, Chen D, et al. MicroRNA-495-3p inhibits multidrug resistance by modulating autophagy through GRP78/mTOR axis in gastric cancer. Cell Death Dis. 2018;9(11):1070.

15. Tan M, Mu X, Liu Z, Tao L, Wang J, Ge J, et al. microRNA-495 promotes bladder cancer cell growth and invasion by targeting phosphatase and tensin homolog. Biochem Biophys Res Commun. 2017;483(2):867-73.

16. Du W, Yin L, Tong P, Chen J, Zhong Y, Huang J, et al. MiR-495 targeting $\mathrm{dvl}-2$ represses the inflammatory response of ankylosing spondylitis. Am J Transl Res. 2019;11(5):2742-53.

17. Yang F, Li XF, Cheng LN, Li XL. Long non-coding RNA CRNDE promotes cell apoptosis by suppressing miR-495 in inflammatory bowel disease. Exp Cell Res. 2019. https://doi.org/10.1016/j.yexcr.2019.06.029.

18. Wang $X$, Jin H, Jiang $S$, Xu Y. MicroRNA-495 inhibits the high glucoseinduced inflammation, differentiation and extracellular matrix accumulation of cardiac fibroblasts through downregulation of NOD1. Cell Mol Biol Lett. 2018;23:23.

19. Dellinger RP, Levy MM, Rhodes A, Annane D, Gerlach H, Opal SM, et al. Surviving Sepsis Campaign: international guidelines for management of severe sepsis and septic shock, 2012. Intensive Care Med. 2013;39(2):165-228.

20. Singer M, Deutschman CS, Seymour CW, Shankar-Hari M, Annane D, Bauer M, et al. The Third International Consensus Definitions for Sepsis and Septic Shock (Sepsis-3). JAMA. 2016;315(8):801-10.

21. Dejager L, Pinheiro I, Dejonckheere E, Libert C. Cecal ligation and puncture: the gold standard model for polymicrobial sepsis? Trends Microbiol. 2011;19(4):198-208.

22. Anand D, Das S, Bhargava S, Srivastava LM, Garg A, Tyagi N, et al. Procalcitonin as a rapid diagnostic biomarker to differentiate between 
culture-negative bacterial sepsis and systemic inflammatory response syndrome: a prospective, observational, cohort study. J Crit Care. 2015;30(1):218 e7-12.

23. Bloos F, Reinhart K. Rapid diagnosis of sepsis. Virulence. 2014;5(1):154-60.

24. Bou Chebl R, El Khuri C, Shami A, Rajha E, Faris N, Bachir R, et al. Serum lactate is an independent predictor of hospital mortality in critically ill patients in the emergency department: a retrospective study. Scand J Trauma Resusc Emerg Med. 2017;25(1):69.

25. Sankar V, Webster NR. Clinical application of sepsis biomarkers. J Anesth 2013;27(2):269-83.

26. Rogobete AF, Sandesc D, Bedreag OH, Papurica M, Popovici SE, Bratu T, et al. MicroRNA expression is associated with sepsis disorders in critically ill polytrauma patients. Cells. 2018;7(12):271.

27. Zhang W, Jia J, Liu Z, Si D, Ma L, Zhang G. Circulating microRNAs as biomarkers for Sepsis secondary to pneumonia diagnosed via Sepsis 3.0. BMC Pulm Med. 2019;19(1):93.

28. Tan A, Luo R, Ruan P. miR-495 promotes apoptosis and inhibits proliferation in endometrial cells via targeting PIK3R1. Pathol Res Pract. 2019;215(3):594-9.

29. Lv L, Wang Q, Yang Y, Ji H. MicroRNA495 targets Notch1 to prohibit cell proliferation and invasion in oral squamous cell carcinoma. Mol Med Rep. 2019;19(1):693-702.

30. Chen $\mathrm{H}$, Wang $X$, Bai J, He A. Expression, regulation and function of miR495 in healthy and tumor tissues. Oncol Lett. 2017;13(4):2021-6.

31. Wang C, Yun Z, Zhao T, Liu X, Ma X. MiR-495 is a predictive biomarker that downregulates GFI1 expression in medulloblastoma. Cell Physiol Biochem. 2015;36(4):1430-9.

32. Li NX, Sun JW, Yu LM. Evaluation of the circulating MicroRNA-495 and Stat3 as prognostic and predictive biomarkers for lower extremity deep venous thrombosis. J Cell Biochem. 2018;119(7):5262-73.
33. Mishra S, Srivastava AK, Suman S, Kumar V, Shukla Y. Circulating miRNAs revealed as surrogate molecular signatures for the early detection of breast cancer. Cancer Lett. 2015;369(1):67-75.

34. Sato R, Kuriyama A, Takada T, Nasu M, Luthe SK. Prevalence and risk factors of sepsis-induced cardiomyopathy: a retrospective cohort study. Medicine (Baltimore). 2016;95(39):e5031.

35. Li Y, Ge S, Peng Y, Chen X. Inflammation and cardiac dysfunction during sepsis, muscular dystrophy, and myocarditis. Burns Trauma. 2013;1(3):109-21.

36. Zhou T, Xiang DK, Li SN, Yang LH, Gao LF, Feng C. MicroRNA-495 ameliorates cardiac microvascular endothelial cell injury and inflammatory reaction by suppressing the NLRP3 inflammasome signaling pathway. Cell Physiol Biochem. 2018;49(2):798-815.

37. Tomita K, Takashina M, Mizuno N, Sakata K, Hattori K, Imura J, et al. Cardiac fibroblasts: contributory role in septic cardiac dysfunction. J Surg Res. 2015;193(2):874-87.

38. Pathan N, Franklin JL, Eleftherohorinou H, Wright VJ, Hemingway CA, Waddell SJ, et al. Myocardial depressant effects of interleukin 6 in meningococcal sepsis are regulated by p38 mitogen-activated protein kinase. Crit Care Med. 2011:39(7):1692-711.

39. Chaudhry H, Zhou J, Zhong Y, Ali MM, McGuire F, Nagarkatti PS, et al. Role of cytokines as a double-edged sword in sepsis. Vivo. 2013;27(6):669-84.

\section{Publisher's Note}

Springer Nature remains neutral with regard to jurisdictional claims in published maps and institutional affiliations.
Ready to submit your research? Choose BMC and benefit from:

- fast, convenient online submission

- thorough peer review by experienced researchers in your field

- rapid publication on acceptance

- support for research data, including large and complex data types

- gold Open Access which fosters wider collaboration and increased citations

- maximum visibility for your research: over 100M website views per year

At BMC, research is always in progress.

Learn more biomedcentral.com/submissions 